\title{
NLRP12 in autoimmune diseases
}

Editorial

\section{Prajwal Gurung and Thirumala-Devi Kanneganti}

Nucleotide-binding oligomerization domain (NOD)like receptors (NLRs) are a group of cytoplasmic sensors that survey danger signals released by invading pathogen or damaged self-tissues. NLRs can be categorized into subgroups based on their ability to form inflammasome, potentiate inflammatory signaling, act as transcriptional regulators and negatively regulate inflammatory pathways. NLRP12, first described about 10 years ago, is a cytoplasmic sensor to be categorized as a negative regulator of inflammation. A recent study has also shown that NLRP12 can form an inflammasome during infection with Yersinia pestis, although this study needs to be substantiated by independent groups [1].

Two seminal studies in 2011 showed that NLRP12 is a crucial negative regulator of NF- $\mathrm{KB}$ signaling pathway $[2,3]$. The absence of NLRP12 in mice resulted in severe uncontrolled inflammation that rendered these NLRP12deficient mice highly susceptible to experimental colitis and inflammation-induced tumorigenesis [2, 3]. NLRP12 was crucial in both hematopoietic and non-hematopoietic compartment for controlling overt inflammation, colitis and colitis-associated tumorigenesis. We have further shown that NLRP12 also negatively regulates NF-кB signaling during Salmonella infection to limit inflammation [4]. While the etiology of infectious diseases, colitis and cancer require coordination of several immune and stromal cells, most of the mechanistic insights on the molecular underpinnings of NLRP12 have only been conducted in innate immune cells that include macrophages and dendritic cells. Specifically, $T$ cells are central to the regulation of all of these disease manifestations but the role of NLRP12 in T cells was not known.

Analysis of NLRP12 expression in various cell populations revealed that $\mathrm{T}$ cells express relatively higher levels of NLRP12 in comparison to both dendritic cells and macrophages [5]. While NLRP12 was not required for negative and positive selection of $\mathrm{T}$ cells in the thymus, our studies showed that Nlrp $12^{-/} \mathrm{T}$ cells produce higher levels of Th1 (IFN- $\gamma$ ), Th2 (IL-4) and Th17 (IL17) cytokines following ex vivo stimulation, suggesting a cell intrinsic role for NLRP12 in negatively regulating $\mathrm{T}$ cell activation [5]. In agreement, in a $\mathrm{T}$ cell transferinduced colitis model, mice receiving Nlrp $12^{-/-} \mathrm{T}$ cells exhibited dramatically severe colitis when compared to mice receiving WT T cells. In addition to severe colitis, mice receiving Nlrp12-1- $\mathrm{T}$ cells also displayed severe inflammation of the skin and ears, i.e. atopic dermatitis.
Further analysis of cytokines in these diseased mice showed that the disease transferring Nlrp $12^{-/} \mathrm{T}$ cells also produced excessive levels of Th2 cytokines such as IL4, IL-5 and IL-13, which are critical in promoting atopic dermatitis. Surprisingly, Nlrp $12^{-/-}$mice showed protection from classical disease symptoms of experimental autoimmune encephalitis (EAE), a $\mathrm{T}$ cell driven autoimmune disease that mimics multiple sclerosis in humans. Closer observation revealed that Nlrp $12^{-/ 2}$ mice suffered from non-classical symptoms of EAE that include loss of balance and ataxia. Interestingly, the atypical EAE phenotype displayed by $\mathrm{Nlrp} 12^{-/-}$mice are symptoms that are more prevalent in humans and could be attributed to excessive IL-4 production by T cells. Thus, Nlrp12mice present a much more realistic model to understand multiple sclerosis in humans. Anti-IL-4 neutralizing antibodies treatment reversed the loss of balance and ataxia symptoms of Nlrp $12^{--}$mice. Mechanistically, we showed that NLRP12 operates downstream of TCRsignaling to negatively regulate the $\mathrm{NF}-\mathrm{kB}$ signaling pathway and inhibit production of all major $\mathrm{T}$ cell cytokines [5]. The importance of our studies is highlighted by human genetic studies where missense mutations in the NLRP12 gene have been reported to result in severe autoinflammatory diseases highlighted by periodic fever syndrome and atopic dermatitis $[6,7]$. Our studies suggest that understanding and targeting $\mathrm{T}$ cells in these patients with NLRP12 mutations might be a potential therapeutic approach. Now that we have shown NLRP12 also plays an important role in EAE, screening human multiple sclerosis patients for mutations in the NLRP12 gene will be important to understand this debilitating autoimmune disease.

Using two different $\mathrm{T}$ cell driven autoimmune disease models ( $\mathrm{T}$ cell driven colitis and EAE), we showed that NLRP12 plays central roles in T cells to control overt inflammation and maintain cellular homeostasis [5]. The exact molecular mechanisms of how NLRP12 tame $\mathrm{NF}-\kappa \mathrm{B}$ activation in T cells will need to be studied. It should be noted that molecular pathways leading to the activation of macrophages/dendritic cells by TLR ligation is completely different from activation of T cells by TCR ligation; yet NLRP12 is able to negatively regulate NF- $\kappa B$ activation in both of these cell types. It will be interesting to examine whether NLRP12 employs similar molecular strategies to block NF- $\mathrm{kB}$ activation in T cells compared to macrophages/dendritic cells. While the use of adoptive transfer, passive transfer and chimeras were invaluable 
in demonstrating T cell intrinsic role for NLRP12 in our studies [5], generation of NLRP12-conditional knockout mice (Nlrp1 $2^{\text {floxflox }}$ mice) will be central in deciphering the precise role of NLRP12 in T cells. The role of NLRP12 in various $\mathrm{T}$ cell subsets (naïve, activated, memory, regulatory) is completely unknown and needs further investigation. Importance of NLRP12 in T cells during acute and chronic infections or vaccine treatments are all open questions that will need to be examined as well. In addition, the role of NLRP12 in other immune cells such as B cells, NK cells and gamma delta $\mathrm{T}$ cells are completely unknown and NLRP 12 conditional mice will be instrumental in these studies. The roles of NLRP12 are only beginning to be uncovered and the complete understanding of how NLRP12 regulates inflammation in various disease settings, immune cells and inflammasomes will undoubtedly lead to novel treatment therapies.

Thirumala-Devi Kanneganti: Department of Immunology, St Jude Children's Research Hospital, Memphis, TN, USA
Correspondence to: Thirumala-Devi Kanneganti, email Thirumala-Devi.Kanneganti@StJude.org

Keywords: Immunology and Microbiology Section, Immune response, Immunity

Received: June 16, 2015

Published: June 22, 2015

\section{REFERENCES}

1. Vladimer GI et al. Immunity. 2012; 37: 96-107.

2. Allen IC et al. Immunity. 2012; 36, 742-754 .

3. Zaki MH et al. Cancer cell. 2011; 20, 649-660.

4. Zaki MH et al. Proc. Natl. Acad. Sci. USA. 2014; 111, 385390.

5. Lukens JR et al. Immunity. 2015; 42, 654-664.

6. Jeru I et al. Proc. Natl. Acad. Sci. USA. 2008; 105, 16141619.

7. Macaluso F et al. Exp. Dermatol. 2007; 16, 692-698. 